\title{
Genome Sequences of COVID-19 from Jordanian Patients in Comparison with the Global Pandemic Strains and the Transmission Route
}

\author{
Ihsan Mahasneh ${ }^{1 *}$, Mustafa Ennaji ${ }^{2}$, Berjas Abumsimir ${ }^{2}$ \\ ${ }^{1}$ Department of Applied Biology, Faculty of Science, University of Sharjah, Sharjah, UAE \\ ${ }^{2}$ Virology Laboratories, Faculty of Sciences and Techniques Mohammedia, Hassan II University of Casablanca, Casablanca, Morocco \\ Email: ^imahasneh@sharjah.ac.ae, lvmqbetb.lab@gmail.com, berjas_last@yahoo.com
}

How to cite this paper: Mahasneh, I., Ennaji, M. and Abumsimir, B. (2021) Genome Sequences of COVID-19 from Jordanian Patients in Comparison with the Global Pandemic Strains and the Transmission Route. Journal of Biosciences and Medicines, 9, 77-93.

https://doi.org/10.4236/jbm.2021.93009

Received: February 12, 2021

Accepted: March 26, 2021

Published: March 29, 2021

Copyright $\odot 2021$ by author(s) and Scientific Research Publishing Inc. This work is licensed under the Creative Commons Attribution International License (CC BY 4.0).

http://creativecommons.org/licenses/by/4.0/

\begin{abstract}
In this research study, we reported a pioneer investigation on the phylogenetic profile of genomic sequences and the prevalence of COVID-19 strains in Jordan; in comparison with global strains, as part of concerted global efforts in combating the COVID-19 pandemic. A total of 28 genome sequences of the hCoV-19/Jordan strain were employed in this study. The phylogenetic analysis of the genome sequences of hCoV-19/Jordan strain, which was conducted on the databases of NCBI and GASAID revealed that the hCoV-19/ Jordan strain belongs to the $\mathrm{G}, \mathrm{V}$, and $\mathrm{S}$ clades with distribution coverage rates of $89 \%, 7 \%$ and $4 \%$, respectively. Comparison of the hCoV-19/Jordan clades with global clades distribution profile showed evidently and conclusively, a unique pandemic profile of multiple geographical sources of COVID-19, imported into Jordan primarily through cryptic means from European countries (UK, Belgium, Sweden, Russia) and Morocco, which are entry points into Jordan. This is due to the reciprocal heavy air traffic route between Jordan and the capitals of these European countries, with Morocco being the primary entry point into Jordan. The results of this study represent a marked addition to investigations on the prevalence rate of the hCovid-19/Jordan strain in comparison to the global strains sequences, and provides a better understanding on comparative diagnosis technique for COVID-19 worldwide. Phylogenetic analysis of the top 100 and top 10 taxonomic trees revealed almost identical homology $(99.98 \%-100 \%)$ between hCoV-19/Jordan strain and the strains that broke out in Wuhan, the MT642226.1. Furthermore, it can be inferred that there was no marked change in the virus mutation that occurred during the period of this study taking into cognizance the fact that there was identical homology $(99.98 \%-100 \%)$ among the 28 sequences of hCoV-19/Jordan strains. Similarly, all sequences of the Jordanian strains of hCoV-19/Jordan
\end{abstract}


were compared to the England and American strains and showed identical homology of (99.98\% - 100\%).

\section{Keywords}

COVID-19 Phylogenetic, COVID-19 in Jordan, Coronavirus SARS-CoV-2, Viral Infection, COVID-19 Pandemic

\section{Introduction}

Since the World Health Organization (WHO) officially declared the outbreak of COVID-19 a pandemic on March 11, 2020, there has been a global growing interest in research targeted at combating the virus due to the surge in infection, as well as the prevalence rate. This has made it imperative and expedient for all probable preventative measures to be adopted in combating the global surge in infection pending the discovery of a vaccine for the virus. Currently, there are over 40 million confirmed cases globally as reported by WHO. The COVID-19 strain responsible for the current global pandemic was identified early this year as the $\beta$-coronavirus ( $\mathrm{CoV})$, which belongs to the Coronaviridae family and it has a single positive-strand RNA genome [1] [2] [3]. This strain-type is among several strains of coronaviruses that are pathogenic to humans; most are associated with mild clinical symptoms, with the exception of the Severe Acute Respiratory Syndrome (SARS) coronavirus (SARS-CoV) and it was reported as a novel $\beta$-coronavirus two decades ago [4] [5] [6]. In 2012, the same strain-type was detected in Saudi Arabia, and since then, it has been known as the Middle East Respiratory Syndrome (MERS) coronavirus (MERS-CoV) [7] [8]. It was later imported into South Korea, and caused about 2500 confirmed cases of infections [9] [10]. In late December, 2019, after global detection of the COVID-19 strain in many countries, the WHO has declared it as a pandemic on March 11, 2020.

A phylogenetic tree with the geographical mutation transmission of SARSCoV-2 has previously been reported to have a genome size of about 30 kilobases, and encodes 10 genes of a single positive-stranded RNA (ribonucleic acid) [1] [2] [11]-[17]. Furthermore, a comprehensive study conducted by the World Health Organization [18] (employing 10,022 SARS-CoV-2 genomes from 68 different countries) showed how the SARS-CoV-2 genome has evolved over the years, spreading to different parts of the world. In total, 65,776 variants and 5775 distinct variants, comprised of the most frequent mutations causing the SARSCoV-2 pandemic were detected by WHO. Additionally, 2969 missense mutations, 1965 synonymous mutations, and 484 mutations in non-coding regions were also discovered by WHO [1]. It was reported by [12] based on data from the public database of the Global Initiative on Sharing All Influenza Data (GISAID) that three major clades of SARS-CoV-2 exists, and have subsequently 
been named as clade G (variant of the spike protein S-D614G), clade V (variant of the ORF3a coding protein NS3-G251), and clade S (variant ORF8L84S).

Jordan and its Mediterranean crossing route, and global tourist attraction centers represent an important route for global spread of COVID-19. The spread of COVID-19 is most likely because of Jordan's heavy air traffic, which is induced by Jordan's route nexus with Europe and many north African countries. It is also attributed to Jordan's close border with Asian countries like China, Pakistan, Nepal, and gulf countries like Saudi, UAE and Bahrain. Up to date of this paper, the COVID-19 pandemic has spread progressively reaching $110,974,862$ confirmed cases of COVID-19, including 2,460,792 deaths worldwide and showed a marked increase in infection in Jordan exceeding 364,000 confirmed cases. Therefore, this paper as the first study aimed at investigating the COVID-19 in Jordan as part of the global pandemic and in comparison to global strains, has a vital role for Jordan as well as other parts of the world for better understanding the COVID-19 pandemic across the world which will enhance the global understanding of the transmission routes as well as the mechanism of action to combating the its pandemic.

\section{Materials and Methods}

\subsection{Jordan National SARS-CoV-2 Pandemic Surveillance Committee}

After the first confirmed case of SARS-CoV-2 infection in Jordan, a National SARS-CoV-2 Pandemic Surveillance Committee was instituted with the National Order number (17) to oversee the SAR-CoV-2 surveillance and combat program.

\subsection{Collection of Clinical Samples and Extraction of RNA Using RT-PCR}

A total of 28 nasopharyngeal swab samples (26 from Amman \& 2 from Irbid) were collected from symptomatic and high index of suspicion patients at the Biolab Diagnostic Laboratories, Amman, Jordan for SAR-CoV-2 investigation using the RT-PCR and RNA extraction technique (in line with the manufacturer's protocol). Quality control assurance measures for SAR-CoV-2 RNA purity were conducted in line with the extraction protocol. The genome of the 28 samples was later dispatched to the Scripps Research Institute (TSRI), La Jolla, California, USA for genome sequencing.

\subsection{Sequencing of the Full Length Genome of Jordanian SAR-CoV-2 Strains}

The (28) genome samples from Jordan (26 from Amman and 2 from Irbid) were subjected to complete genome sequencing in the Scripps Research Institute (TSRI), La Jolla, California, USA as previously described [11]. The resultant se- 
quences were later deposited by the Scripps Research Institute (TSRI) into the databases of the NCBI GenBank (http://www.ncbi.nlm.nih.gov/) and Global Initiative on Sharing All Influenza Data (GISAID) (http://www.gisaid.org/) employing the tag hCoV-19/Jordan vis-à-vis each respective accession number (see Table 1 for details of the accession numbers and other descriptions).

\subsection{Using GISAID (Global Initiative on Sharing All Influenza) Public Domain Databank for Phylogenetic Comparison with COVID-19 Sequences of Global Strains}

For comparison of our strains sequences of the COVID-19 with the genomic sequences of the global strains from different countries, we have used with acknowledgments the COVID-19 sequences available on the Public Domain Databank platform of GISAID and EpiFlu ${ }^{\text {tix }}$ database, as hosted by the Federal Republic of Germany; which provides us with rapid sharing of COVID-19 sequences enabling us for rapid and conclusive global comparison on the epidemic and pandemic of the COVID-19 (http://www.gisaid.org/).

Sequence alignment was conducted using human coronavirus sequences registered in the Global Initiative on Sharing All Influenza Data (GISAID) and NCBI GenBank. Using the available sequences and the software on the Public Domain of the GISAID (http://www.gisaid.org/) and the NCBI

(https://blast.ncbi.nlm.nih.gov); the Phylogenetic trees were constructed employing for Neighbor Joining (NJ); Minimum Evaluation Analysis and the NJ-Taxonomic Distance Tree. The accession numbers of the 28 sequences of hCoV-19/Jordan were used to construct the phylogenetic tree in comparison with the closest sequences of the top 100 and top 10 taxonomic trees, and (99.98\% - 100\%) semblance to those sequences available on the NCBI (https://blast.ncbi.nlm.nih.gov). In this comparative study, we selected a set of sequences from different parts of the world. Sequences were selected from Europe (3 from United Kingdom, 3 from Belgium, 2 from Sweden, and 3 from Russia), Asia (9 from Pakistan, 1 from Nepal, 9 from Bahrain, and 4 from Lebanon) and Africa (10 from Morocco) because of the geographical limitrophe of Jordan with these countries. Sequences were assembled and mapped to the SARS-CoV-2 that are available on the Public Domain of the GISAID (http://www.gisaid.org/) and the NCBI (https://blast.ncbi.nlm.nih.gov).

\section{Results}

The phylogenetic tree of the hCoV-19/Jordan sequences in comparison with selected global sequences is presented in Figure 1 which shows almost identical homology $(99.98 \%$ - 100\%) with the top 100 and top 10 taxonomic trees. The results showed that the spreading profile in Jordan is composed of (5) geographical clusters vis-à-vis the origin of the pandemic. These include 2 different clusters similar to sequences from the UK, 1 cluster from Morocco and 2 clusters with local transmission designation. The neighbor joining (NJ) of distance-based 
Table 1. Sequences description for the Covid-2 of the genus Betacoronavirus and family Coronaviridae; during pandemic period of March $1^{\text {st }}$. and October $1^{\text {st }} .2020$ as compared with selected global strains.

\begin{tabular}{|c|c|c|c|c|}
\hline No & $\begin{array}{l}\text { GeneBank/Sequence } \\
\text { accession number }\end{array}$ & $\begin{array}{l}\text { Submission } \\
\text { date }\end{array}$ & $\begin{array}{l}\text { Sequence } \\
\text { length }\end{array}$ & Geographical location \\
\hline 1 & MW041156 & $2020-09-28$ & 29,870 & United Kingdom \\
\hline 2 & MW031799 & $2020-09-23$ & 29,903 & Pakistan \\
\hline 3 & MW031800 & $2020-09-23$ & 29,903 & Pakistan \\
\hline 4 & MW031801 & $2020-09-23$ & 29,903 & Pakistan \\
\hline 5 & MW031802 & $2020-09-23$ & 29,903 & Pakistan \\
\hline 6 & MW031803 & $2020-09-23$ & 29,903 & Pakistan \\
\hline 7 & MT890462 & 2020-08-17 & 29,903 & Russia: Moscow \\
\hline 8 & MT879619 & $2020-08-13$ & 29,897 & Pakistan \\
\hline 9 & MT873892 & $2020-08-11$ & 29,821 & United Kingdom \\
\hline 10 & MT811544 & 2020-07-29 & 29,409 & Jordan: Amman \\
\hline 11 & MT811545 & $2020-07-29$ & 29,409 & Jordan: Amman \\
\hline 12 & MT811546 & $2020-07-29$ & 29,409 & Jordan: Amman \\
\hline 13 & MT811548 & $2020-07-29$ & 29,409 & Jordan: Amman \\
\hline 14 & MT811549 & $2020-07-29$ & 29,409 & Jordan: Amman \\
\hline 15 & MT811550 & $2020-07-29$ & 29,409 & Jordan: Amman \\
\hline 16 & MT811551 & 2020-07-29 & 29,409 & Jordan: Amman \\
\hline 17 & MT811552 & $2020-07-29$ & 29,412 & Jordan: Amman \\
\hline 18 & MT811553 & 2020-07-29 & 29,409 & Jordan: Amman \\
\hline 19 & MT811554 & $2020-07-29$ & 29,409 & Jordan: Amman \\
\hline 20 & MT811555 & 2020-07-29 & 29,409 & Jordan: Amman \\
\hline 21 & MT811556 & $2020-07-29$ & 29,409 & Jordan: Amman \\
\hline 22 & MT811557 & $2020-07-29$ & 29,409 & Jordan: Amman \\
\hline 23 & MT811558 & 2020-07-29 & 29,409 & Jordan: Amman \\
\hline 24 & MT811560 & $2020-07-29$ & 29,409 & Jordan: Irbid \\
\hline 25 & MT811561 & 2020-07-29 & 29,409 & Jordan: Amman \\
\hline 26 & MT811562 & $2020-07-29$ & 29,409 & Jordan: Irbid \\
\hline 27 & MT811564 & 2020-07-29 & 29,409 & Jordan: Amman \\
\hline 28 & MT811565 & 2020-07-29 & 29,409 & Jordan: Amman \\
\hline 29 & MT811566 & 2020-07-29 & 29,406 & Jordan: Amman \\
\hline 30 & MT811567 & 2020-07-29 & 29,409 & Jordan: Amman \\
\hline 31 & MT811568 & 2020-07-29 & 29,409 & Jordan: Amman \\
\hline 32 & MT811569 & $2020-07-29$ & 29,409 & Jordan: Amman \\
\hline 33 & MT801000 & $2020-07-27$ & 29,854 & Lebanon \\
\hline 34 & MT801001 & 2020-07-27 & 29,829 & Lebanon \\
\hline 35 & MT801002 & $2020-07-27$ & 29,865 & Lebanon \\
\hline 36 & MT801003 & $2020-07-27$ & 29,779 & Lebanon \\
\hline 37 & MT789712 & $2020-07-22$ & 29,856 & Bahrain \\
\hline
\end{tabular}




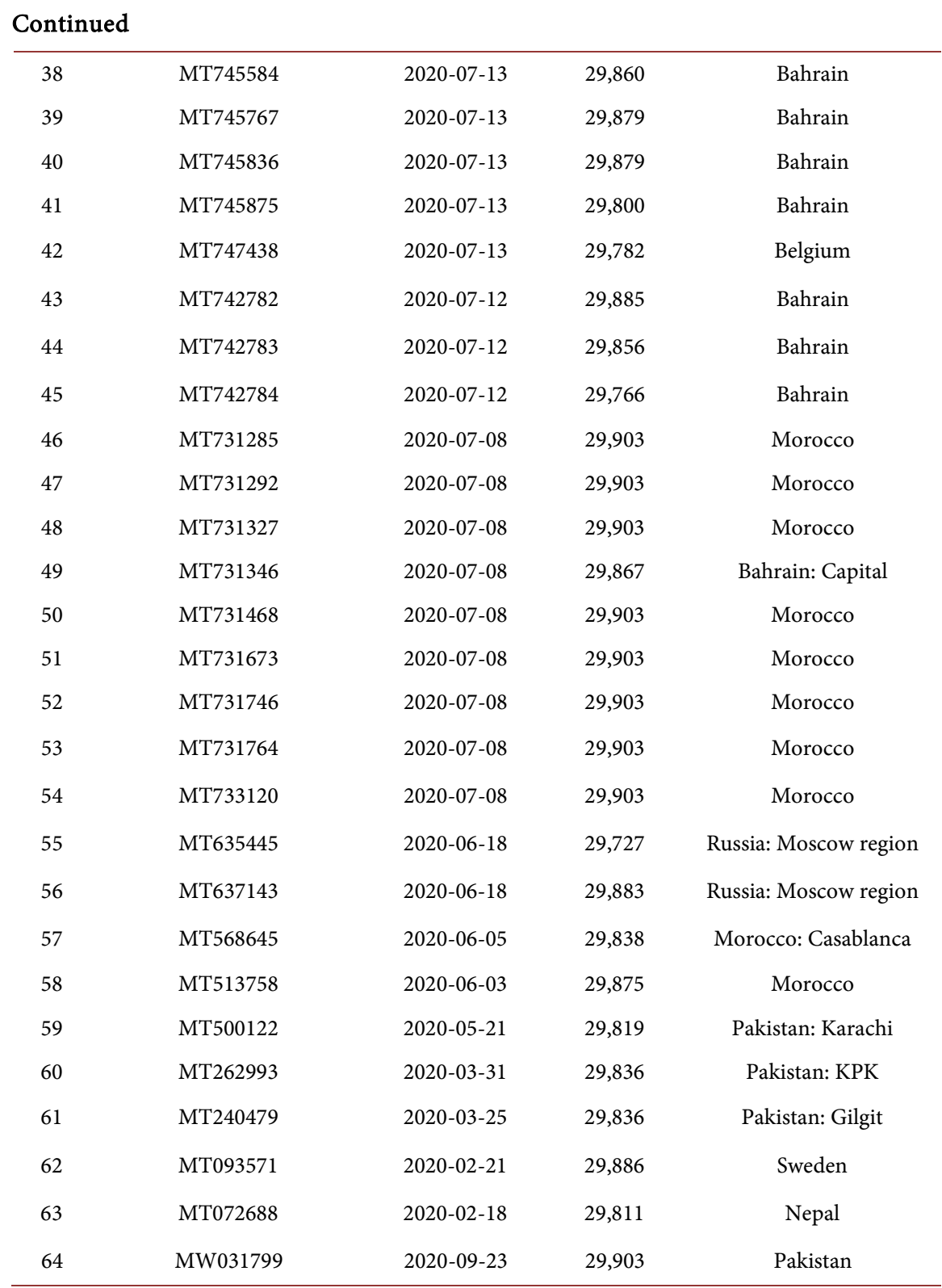

phylogenetic analysis of the top 100 (Figure 2) and top 10 (Figure 3) taxonomic trees revealed almost identical homology between hCoV-19/Jordan strain and the strains that broke out in Wuhan, the MT642226.1.

The sequence description of the hCoV-19/Jordan strain in comparison with global sequences homology $(99.98 \%$ - 100\%) based on NCBI database filter results is presented in Table 1. All sequences of the hCoV-19/Jordan strain were complete, exceeding $(29,400 \mathrm{bp})$, and example of the complete sequence of the hCoV-19/Jordan is given in Figure 3, which were therefore compared with similar complete global sequences. The clades and sub-clades descriptions of the genealogical lineage of the $\mathrm{hCoV}-19 /$ Jordan strain are presented in Table 2; which shows that the hCoV-19/Jordan strain belongs to the G, V, and S clades with spreading frequencies of $89 \%, 7 \%$ and $4 \%$, respectively. 


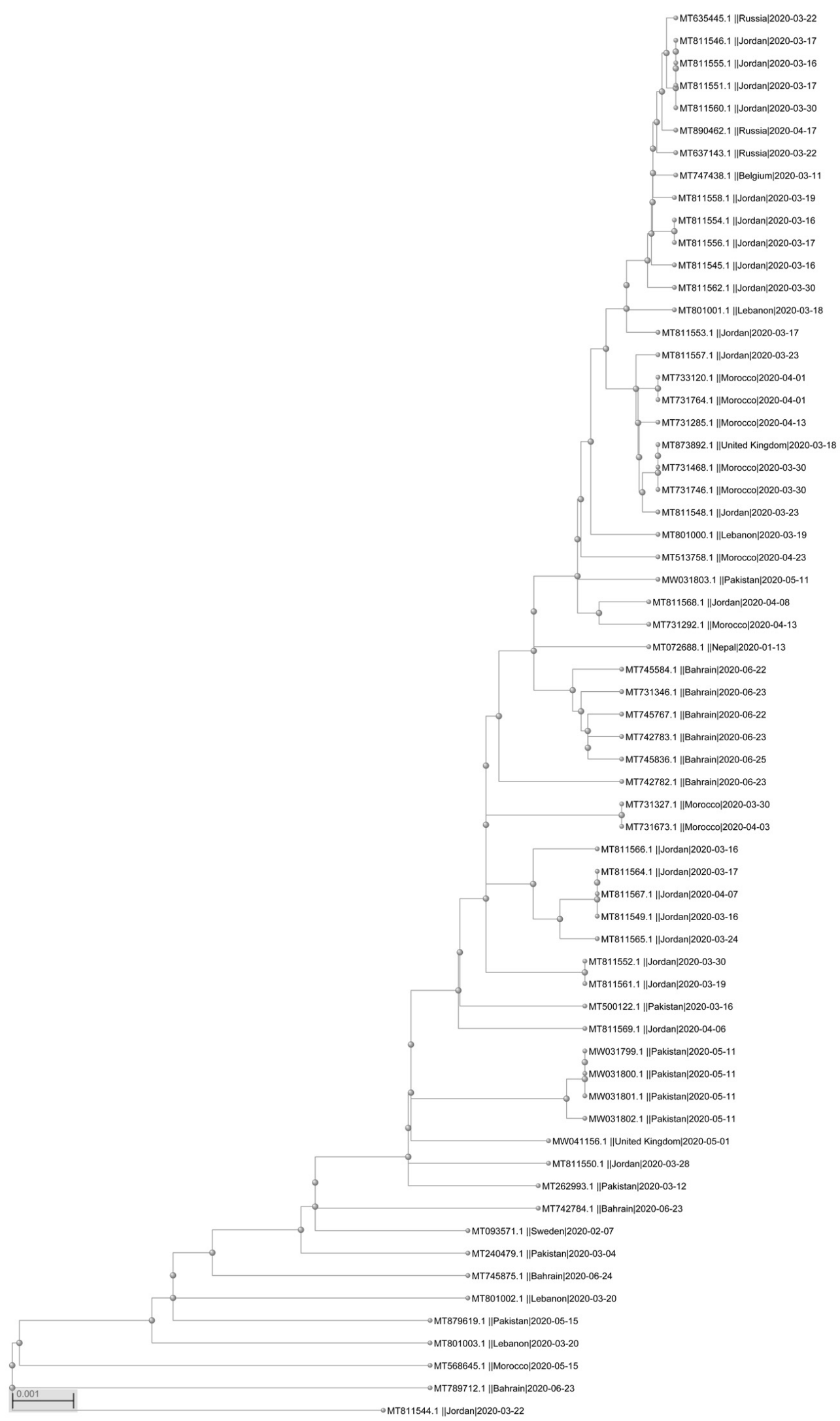

Figure 1. NJ-distance phylogenetic of the hCoV-19/Jordan sequences as compared to the global sequences. 
Severe acute respiratory syndrome coronavirus 2 isolate SARS-CoV-2/human/JOR/SEARCH-0054-JOR/2020, complete g. - Severe acute respiratory syndrome coronavirus 2 isolate SARS-CoV-2/human/JOR/SEARCH-0054-JOR/2020, complete g. - Severe acute respiratory syndrome coronavirus 2 isolate NORW-EBA67 genome assembly, chromosome: 1 Severe acute respiratory syndrome coronavirus 2 isolate NORW-EBD7D genome assembly, chromosome: 1 OSevere acute respiratory syndrome coronavirus 2 isolate SARS-CoV-2/human/USA/CT-UW-651/2020, complete genome 8 Severe acute respiratory syndrome coronavirus 2 isolate SARS-CoV-2/human/USA/WA-UW213/2020, complete genome Severe acute respiratory syndrome coronavirus 2 isolate SARS-CoV-2/human/AUS/VIC860/2020, complete genome - Severe acute respiratory syndrome coronavirus 2 isolate SARS-CoV-2/human/USA/MA-QDX-80/2020, complete genome OSevere acute respiratory syndrome coronavirus $2 \mathrm{hCoV}-19 / \mathrm{Japan} / \mathrm{P} 4-4 / 2020 \mathrm{RNA}$, complete genome viruses $\mid 4$ leaves

Uiruses $\mid 5$ leaves

8 Severe acute respiratory syndrome coronavirus 2 isolate SARS-CoV-2/human/USA/NY-QDX-106/2020, complete genome viruses 7 leaves

8 Severe acute respiratory syndrome coronavirus $2 \mathrm{hCoV}-19 / \mathrm{Japan} / \mathrm{P4}-2 / 2020 \mathrm{RNA}$, complete genome

4 viruses $\mid 4$ leaves

viruses $\mid 5$ leaves

8 Severe acute respiratory syndrome coronavirus 2 isolate SARS-CoV-2/human/SAU/86327/2020, complete genome

4 viruses $\mid 7$ leaves

$\checkmark$ viruses 29 leaves

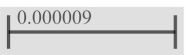

8

iruses | 11 leaves

4

iruses $\mid 17$ leaves

Figure 2. NJ-distance phylogenetic of the hCoV-19/Jordan sequences as compared to the top-100 hits of global sequences.

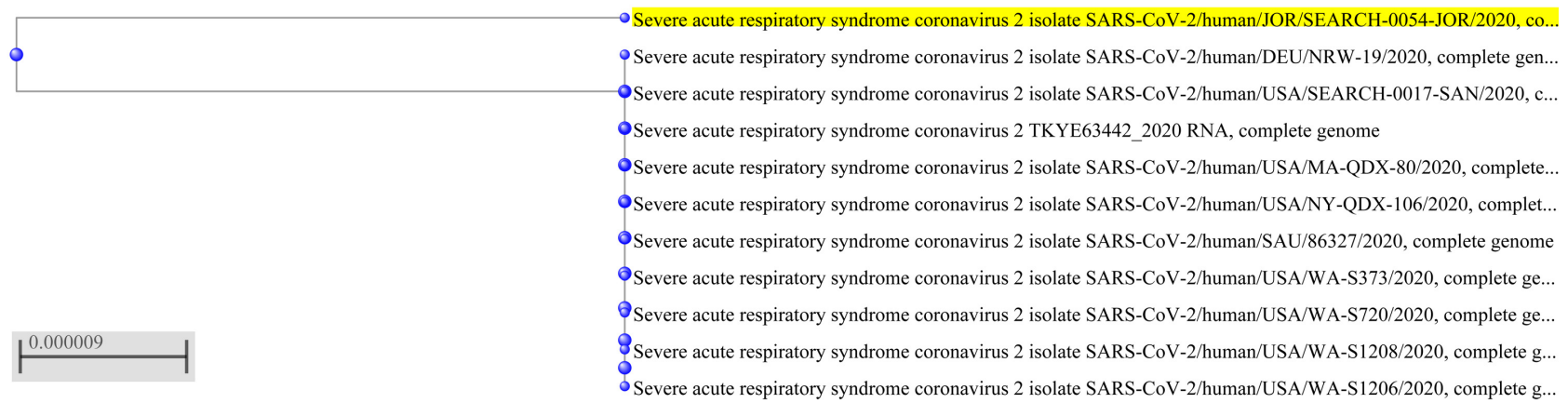

Figure 3. NJ-distance phylogenetic of the hCoV-19/Jordan sequences as compared to the top-10 hits of global sequences.

\section{Discussion}

The phylogenetic analysis of the hCoV-19/Jordan strain constructed from selected global genomes deposited into GISAID for comparison purposes revealed that the 28 genome sequences from Jordan employed in this study were compatible with the evolutionary tree of SARS-CoV-2, and had a complete genome length of $29,400 \mathrm{bp}$ and homology of (99.98\% - 100\%) (Figure 1). This provides a conclusive evidence that the hCoV-19/Jordan is part of the global pandemic strains of SARS-CoV-2. The results of our study showed that the genome length and homology of the hCoV-19/Jordan strain are in consonance with those reported for the SARS-CoV-2 strains globally [1] [3] [11]. The neighbor joining phylogenetic analysis of the top 100 (Figure 2) and top 10 taxonomic trees (Figure 3) showed conclusively that the hCoV-19/Jordan strain had almost identical homology (99.98\% - 100\%) with the strains that broke out in Wuhan, the MT642226.1. This suggests that it is the most recent strain, and has a common ancestry with the strains transmitted and detected in Jordan. Similarly, all sequences of the Jordanian strains of hCoV-19/Jordan were compared to the England and American strains and showed identical homology of (99.98\% - 
Table 2. Characterization of the clades \& genealogical-lineages of the COVID-19 strains clusters during pandemic in Jordan.

\begin{tabular}{|c|c|c|c|c|c|c|c|}
\hline No & $\begin{array}{l}\text { Clade/ } \\
\text { Lineage }\end{array}$ & Virus name & Accession ID & Collection date & Submission date & Length & Geographical region \\
\hline 1 & G/B.1.1 & hCoV-19/Jordan/SR-055/2020 & EPI_ISL_430013 & 2020-04-02 & 2020-04-24 & 29,409 & Asia/Jordan/Amman \\
\hline 2 & & hCoV-19/Jordan/SR-049/2020 & EPI_ISL_430007 & 2020-03-19 & $2020-04-24$ & 29,409 & Asia/Jordan/Amman \\
\hline 3 & & hCoV-19/Jordan/SR-048/2020 & EPI_ISL_430006 & $2020-03-23$ & $2020-04-24$ & 29,409 & Asia/Jordan/Amman \\
\hline 4 & & hCoV-19/Jordan/SR-047/2020 & EPI_ISL_430005 & $2020-03-26$ & $2020-04-24$ & 29,397 & Asia/Jordan/Amman \\
\hline 5 & & hCoV-19/Jordan/SR-046/2020 & EPI_ISL_430004 & 2020-03-17 & $2020-04-24$ & 29,409 & Asia/Jordan/Amman \\
\hline 6 & & hCoV-19/Jordan/SR-045/2020 & EPI_ISL_430003 & 2020-03-16 & $2020-04-24$ & 29,409 & Asia/Jordan/Amman \\
\hline 7 & & hCoV-19/Jordan/SR-044/2020 & EPI_ISL_430002 & $2020-03-16$ & 2020-04-24 & 29,409 & Asia/Jordan/Amman \\
\hline 8 & & hCoV-19/Jordan/SR-043/2020 & EPI_ISL_430001 & 2020-03-17 & $2020-04-24$ & 29,409 & Asia/Jordan/Amman \\
\hline 9 & & hCoV-19/Jordan/SR-041/2020 & EPI_ISL_429999 & 2020-03-17 & $2020-04-24$ & 29,409 & Asia/Jordan/Amman \\
\hline 10 & & hCoV-19/Jordan/SR-036/2020 & EPI_ISL_429996 & $2020-03-23$ & $2020-04-24$ & 29,409 & Asia/Jordan/Amman \\
\hline 11 & & hCoV-19/Jordan/SR-034/2020 & EPI_ISL_429994 & 2020-03-17 & $2020-04-24$ & 29,409 & Asia/Jordan/Amman \\
\hline 12 & & hCoV-19/Jordan/SR-033/2020 & EPI_ISL_429993 & 2020-03-16 & $2020-04-24$ & 29,409 & Asia/Jordan/Amman \\
\hline 13 & & hCoV-19/Jordan/SR-032/2020 & EPI_ISL_429992 & 2020-03-22 & $2020-04-24$ & 29,409 & Asia/Jordan/Amman \\
\hline 14 & G/B.1.1 & hCoV-19/Jordan/SR-054/2020 & EPI_ISL_430012 & 2020-03-30 & $2020-04-24$ & 29,409 & Asia/Jordan/Irbid \\
\hline 15 & G/B.1.1 & hCoV-19/Jordan/SR-052/2020 & EPI_ISL_430009 & $2020-03-30$ & $2020-04-24$ & 29,409 & Asia/Jordan/Irbid \\
\hline 16 & GH/B.1.36 & hCoV-19/Jordan/SR-0335/2020 & EPI_ISL_450187 & 2020-04-08 & $2020-05-20$ & 29,864 & Asia/Jordan/Amman \\
\hline 17 & B.2.1(O) & hCoV-19/Jordan/SR-084/2020 & EPI_ISL_434516 & $2020-03-16$ & $2020-04-29$ & 29,406 & Asia/Jordan/Amman \\
\hline 18 & & hCoV-19/Jordan/SR-057/2020 & EPI_ISL_430015 & $2020-03-24$ & $2020-04-24$ & 29,409 & Asia/Jordan/Amman \\
\hline 19 & & hCoV-19/Jordan/SR-056/2020 & EPI_ISL_430014 & 2020-03-17 & 2020-04-24 & 29,409 & Asia/Jordan/Amman \\
\hline 20 & & hCoV-19/Jordan/SR-053/2020 & EPI_ISL_430011 & 2020-03-19 & 2020-04-24 & 29,409 & Asia/Jordan/Amman \\
\hline 21 & & hCoV-19/Jordan/SR-051/2020 & EPI_ISL_430008 & 2020-04-04 & $2020-04-24$ & 29,409 & Asia/Jordan/Amman \\
\hline 22 & & hCoV-19/Jordan/SR-042/2020 & EPI_ISL_430000 & 2020-03-30 & $2020-04-24$ & 29,412 & Asia/Jordan/Amman \\
\hline 23 & & hCoV-19/Jordan/SR-039/2020 & EPI_ISL_429998 & $2020-03-28$ & $2020-04-24$ & 29,409 & Asia/Jordan/Amman \\
\hline 24 & & hCoV-19/Jordan/SR-037/2020 & EPI_ISL_429997 & 2020-03-16 & $2020-04-24$ & 29,409 & Asia/Jordan/Amman \\
\hline 25 & & hCoV-19/Jordan/SR-035/2020 & EPI_ISL_429995 & $2020-03-30$ & $2020-04-24$ & 29,412 & Asia/Jordan/Amman \\
\hline 26 & S/A & hCoV-19/Jordan/SR-0336/2020 & EPI_ISL_450188 & 2020-04-06 & $2020-05-20$ & 29,864 & Asia/Jordan/Amman \\
\hline 27 & V/B.2 & hCoV-19/Jordan/SR-0338/2020 & EPI_ISL_450189 & 2020-04-06 & $2020-05-20$ & 29,883 & Asia/Jordan/Amman \\
\hline 28 & V/B.2 & hCoV-19/Jordan/SR-0333/2020 & EPI_ISL_450186 & 2020-04-07 & $2020-05-20$ & 29,864 & Asia/Jordan/Amman \\
\hline
\end{tabular}

100\%) (Figure 2 and Figure 3) and as it can be seen from the example of the complete sequence of the hCoV-19/Jordan strain as given in Figure 4.

Comparison of the clades of hCoV-19/Jordan with that of SARS-CoV-2 showed that the most plausible transmission routes are European countries (UK, Belgium, Sweden, Russia) and Morocco, which are entry points into Jordan. This can be attributed to the fact that both the $\mathrm{G}$ and $\mathrm{V}$ clades reported in this study for hCoV-19/Jordan strain are similar to those reported earlier in these European countries [12] [19]. This further suggests that the strain was transmitted through the heavy air traffic transmission rout between EU countries-Morocco-Jordan) after the strains were transmitted from China to EU countries. This is 
>MT811562.1:1-13203,13203-21290 Severe acute respiratory syndrome coronavirus 2 isolate SARS-CoV-2/human/JOR/SEARCH-0054-JOR/2020, complete genome

ATGGAGAGCCTTGTCCCTGGTTTCAACGAGAAAACACACGTCCAACTCAGTTTGCCTGTTTTACAGGTTC GCGACGTGCTCGTACGTGGCTTTGGAGACTCCGTGGAGGAGGTCTTATCAGAGGCACGTCAACATCTTAA AGATGGCACTTGTGGCTTAGTAGAAGTTGAAAAAGGCGTTTTGCCTCAACTTGAACAGCCCTATGTGTTC ATCAAACGTTCGGATGCTCGAACTGCACCTCATGGTCATGTTATGGTTGAGCTGGTAGCAGAACTCGAAG GCATTCAGTACGGTCGTAGTGGTGAGACACTTGGTGTCCTTGTCCCTCATGTGGGCGAAATACCAGTGGC TTACCGCAAGGTTCTTCTTCGTAAGAACGgTAATAAAGGAGCTGGTGGCCATAGTTACGGCGCCGATCTA AAGTCATTTGACTTAGgCGACGAGCTTGGCACTGATCCTTATGAAGATTTTCAAGAAAACTGGAACACTA AACATAGCAGTGGTGTTACCCGTGAACTCATGCGTGAGCTTAACGGAGGGGCATACACTCGCTATGTCGA TAACAACTTCTGTGGCCCTGATGGCTACCCTCTTGAGTGCATTAAAGACCTTCTAGCACGTGCTGGTAAA GCTTCATGCACTTTGTCCGAACAACTGGACTTTATTGACACTAAGAGGGGTGTATACTGCTGCCGTGAAC ATGAGCATGAAATGCTTGGTACACGGAACGTTCTGAAAAGAGCTATGAATTGCAGACACCTTTTGAAAT TAAATTGGCAAAGAAATTTGACACCTTCAATGGGGAATGTCCAAATTTTGTATTTCCCTTAAATTCCATA ATCAAGACTATTCAACCAAGGGTTGAAAAGAAAAAGCTTGATGGCTTTATGGGTAGAATTCGATCTGTCT ATCCAGTTGCGTCACCAAATGAATGCAACCAAATGTGCCTTTCAACTCTCATGAAGTGTGATCATTGTGG TGAAACTTCATGGCAGACGGGCGATTTTGTTAAAGCCACTTGCGAATTTTGTGGCACTGAGAATTTGACT AAAGAAGGTGCCACTACTTGTGGTTACTTACCCCAAAATGCTGTTGTTAAAATTTATTGTCCAGCATGTC ACAATTCAGAAGTAGGACCTGAGCATAGTCTTGCCGAATACCATAATGAATCTGGCTTGAAAACCATTCT TCGTAAGGGTGGTCGCACTATTGCCTTTGGAGGCTGTGTGTTCTCTTATGTTGGTTGCCATAACAAGTGT GCCTATTGGGTTCCACGTGCTAGCGCTAACATAGGTTGTAACCATACAGGTGTTGTTGGAGAAGGTTCCG AAGGTCTTAATGACAACCTTCTTGAAATACTCCAAAAAGAGAAAGTCAACATCAATATTGTTGGTGACTT TAAACTTAATGAAGAGATCGCCATTATTTTGGCATCTTTTTCTGCTTCCACAAGTGCTTTTGTGGAAACT GTGAAAGGTTTGGATTATAAAGCATTCAAACAAATTGTTGAATCCTGTGGTAATTTTAAAGTTACAAAAG GAAAAGCTAAAAAAGGTGCCTGGAATATTGGTGAACAGAAATCAATACTGAGTCCTCTTTATGCATTTGC ATCAGAGGCTGCTCGTGTTGTACGATCAATTTTCTCCCGCACTCTTGAAACTGCTCAAAATTCTGTGCGT GTTTTACAGAAGGCCGCTATAACAATACTAGATGGAATTTCACAGTATTCACTGAGACTCATTGATGCTA TGATGTTCACATCTGATTTGGCTACTAACAATCTAGTTGTAATGGCCTACATTACAGGTGGTGTTGTTCA GTTGACTTCGCAGTGGCTAACTAACATCTTTGGCACTGTTTATGAAAAACTCAAACCCGTCCTTGATTGG CTTGAAGAGAAGTTTAAGGAAGGTGTAGAGTTTCTTAGAGACGGTTGGGAAATTGTTAAATTTATCTCAA CCTGTGCTTGTGAAATTGTCGGTGGACAAATTGTCACCTGTGCAAAGGAAATTAAGGAGAGTGTTCAGAC ATTCTTTAAGCTTGTAAATAAATTTTGGCTTTGTGTGCTGACTCTATCATTATTGGTGGAGCTAAACTT AAAGCCTTGAATTTAGGTGAAACATTTGTCACGCACTCAAAGGGATTGTACAGAAAGTGTGTTAAATCCA GAGAAGAAACTGGCCTACTCATGCCTCTAAAAGCCCCAAAAGAAATTATCTTCTTAGAGGGAGAAACACT TCCCACAGAAGTGTTAACAGAGGAAGTTGTCTTGAAAACTGGTGATTTACAACCATTAGAACAACCTACT AGTGAAGCTGTTGAAGCTCCATTGGTTGGTACACCAGTTTGTATTAACGGGCTTATGTTGCTCGAAATCA AAGACACAGAAAAGTACTGTGCCCTTGCACCTAATATGATGGTAACAAACAATACCTTCACACTCAAAGG CGGTGCACCAACAAAGGTTACTTTTGGTGATGACACTGTGATAGAAGTGCAAGGTTACAAGAGTGTGAAT ATCACTTTTGAACTTGATGAAAGGATTGATAAAGTACTTAATGAGAAGTGCTCTGCCTATACAGTTGAAC TCGGTACAGAAGTAAATGAGTTCGCCTGTGTTGTGGCAGATGCTGTCATAAAAACTTTGCAACCAGTATC TGAATTACTTACACCACTGGGCATTGATTTAGATGAGTGGAGTATGGCTACATACTACTTATTTGATGAG TCTGGTGAGTTTAAATTGGCTTCACATATGTATTGTTCTTTTTACCCTCCAGATGAGGATGAAGAAGAAG GTGATTGTGAAGAAGAAGAGTTTGAGCCATCAACTCAATATGAGTATGGTACTGAAGATGATTACCAAGG TAAACCTTTGGAATTGGTGCCACTTCTGCTGCTCTTCAACCTGAAGAAGAGCAAGAAGAAGATTGGTTA GATGATGATAGTCAACAAACTGTTGGTCAACAAGACGGCAGTGAGGACAATCAGACAACTACTATTCAAA CAATTGTTGAGGTTCAACCTCAATTAGAGATGGAACTTACACCAGTTGTTCAGACTATTGAAGTGAATAG TTTTAGTGGTTATTTAAAACTTACTGACAATGTATACATTAAAAATGCAGACATTGTGGAAGAAGCTAAA AAGGTAAAACCAACAGTGGTTGTTAATGCAGCCAATGTTTACCTTAAACATGGAGGAGGTGTTGCAGGAG CCTTAAATAAGGCTACTAACAATGCCATGCAAGTTGAATCTGATGATTACATAGCTACTAATGGACCACT TAAAGTGGGTGGTAGTTGTGTTTTAAGCGGACACAATCTTGCTAAACACTGTCTTCATGTTGTCGGCCCA AАTGTTAACAAAGGTGAAGACATTCAACTTCTTAAGAGTGCTTATGAAAATTTTAATCAGCACGAAGTTC TACTTGCACCATTATTATCAGCTGGTATTTTTGGTGCTGACCCTATACATTCTTTAAGAGTTTGTGTAGA TACTGTTCGCACAAATGTCTACTTAGCTGTCTTTGATAAAAATCTCTATGACAAACTTGTTTCAAGCTTT TTGGAAATGAAGAGTGAAAAGCAAGTTGAACAAAAGATCGCTGAGATTCCTAAAGAGGAAGTTAAGCCAT TTATAACTGAAAGTAAACCTTCAGTTGAACAGAGAAAACAAGATGATAAGAAAATCAAAGCTTGTGTTGA AGAAGTTACAACAACTCTGGAAGAAACTAAGTTCCTCACAGAAAACTTGTTACTTTATATTGACATTAAT GGCAАTCTTCATCCAGATTCTGCCACTCTTGTTAGTGACATTGACATCACTTTCTTAAAGAAAGATGCTC 
CATATATAGTGGGTGATGTTGTTCAAGAGGGTGTTTTAACTGCTGTGGTTATACCTACTAAAAAGGCTGG TGGCACTACTGAAATGCTAGCGAAAGCTTTGAGAAAAGTGCCAACAGACAATTATATAACCACTTACCCG GGTCAGGGTTTAAATGGTTACACTGTAGAGGAGGCAAAGACAGTGCTTAAAAAGTGTAAAAGTGCCTTTT АСАTTCTACCATCTATTATCTCTAATGAGAAGCAAGAAATTCTTGGAACTGTTTCTTGGAATTTGCGAGA AATGCTTGCACATGCAGAAGAAACACGCAAATTAATGCCTGTCTGTGTGGAAACTAAAGCCATAGTTTCA ACTATACAGCGTAAATATAAGGGTATTAAAATACAAGAGGGTGTGGTTGATTATGGTGCTAGATTTTACT TTTACACCAGTAAAACAACTGTAGCGTCACTTATCAACACACTTAACGATCTAAATGAAACTCTTGTTAC AATGCCACTTGGCTATGTAACACATGGCTTAAATTGGAAGAAGCTGCTCGGTATATGAGATCTCTCAAA GTGCCAGCTACAGTTTCTGTTTCTTCACCTGATGCTGTTACAGCGTATAATGGTTATCTTACTTCTTCTT CTAAAACACCTGAAGAACATTTTATTGAAACCATCTCACTTGCTGGTTCCTATAAAGATTGGTCCTATTC TGGACAATCTACACAACTAGGTATAGAATTTCTTAAGAGAGGTGATAAAAGTGTATATTACACTAGTAAT CCTACCACATTCCACCTAGATGGTGAAGTTATCACCTTTGACAATCTTAAGACACTTCTTTCTTTGAGAG AAGTGAGGACTATTAAGGTGTTTACAACAGTAGACAACATTAACCTCCACACGCAAGTTGTGGACATGTC AATGACATATGGACAACAGTTTGGTCCAACTTATTTGGATGGAGCTGATGTTACTAAAATAAAACCTCAT AАTTCACATGAAGGTAAAACATTTTATGTTTTACCTAATGATGACACTCTACGTGTTGAGGCTTTTGAGT ACTACCACACAACTGATCCTAGTTTTCTGgGTAGGTACATGTCAGCATTAAATCACACTAAAAAGTGGAA ATACCCACAAGTTAATGGTTTAACTTCTATTAAATGGGCAGATAACAACTGTTATCTTGCCACTGCATTG TTAACACTCCAACAAATAGAGTTGAAGTTTAATCCACCTGCTCTACAAGATGCTTATTACAGAGCAAGGG CTGGTGAAGCTGCTAACTTTTGTGCACTTATCTTAGCCTACTGTAATAAGACAGTAGGTGAGTTAGGTGA TGTTAGAGAAACAATGAGTTACTTGTTTCAACATGCCAATTTAGATTCTTGCAAAAGAGTCTTGAACGTG GTGTGTAAAACTTGTGGACAACAGCAGACAACCCTTAAGGGTGTAGAAGCTGTTATGTACATGGGCACAC TTTCTTATGAACAATTAAGAAAGGTGTTCAGATACCTTGTACGTGTGGTAAACAAGCTACAAAATATCT AGTACAACAGGAGTCACCTTTTGTTATGATGTCAGCACCACCTGCTCAGTATGAACTTAAGCATGGTACA TTTACTTGTGCTAGTGAGTACACTGGTAATTACCAGTGTGGTCACTATAAACATATAACTTCTAAAGAAA CTTTGTATTGCATAGACGGTGCTTTACTTACAAAGTCCTCAGAATACAAAGGTCCTATTACGGATGTTTT CTACAAAGAAAACAGTTACACAACAACCATAAAACCAGTTACTTATAAATTGGATGGTGTTGTTTGTACA GAAATTGACCCTAAGTTGGACAATTATTATAAGAAAGACAATTCTTATTTCACAGAGCAACCAATTGATC TTGTACCAAACCAACCATATCCAAACGCAAGCTTCGATAATTTTAAGTTTGTATGTGATAATATCAAATT TGCTGATGATTTAAACCAGTTAACTGGTTATAAGAAACCTGCTTCAAGAGAGCTTAAAGTTACATTTTTC CCTGACTTAAATGGTGATGTGGTGGCTATTGATTATAAACACTACACACCCTCTTTTAAGAAAGGAGCTA AАTTGTTACATAAACCTATTGTTTGGCATGTTAACAATGCAACTAATAAAGCCACGTATAAACCAAATAC CTGGTGTATACGTTGTCTTTGGAGCACAAAACCAGTTGAAACATCAAATTCGTTTGATGTACTGAAGTCA GAGGACGCGCAGGGAATGGATAATCTTGCCTGCGAAGATCTAAAACCAGTCTCTGAAGAAGTAGTGGAAA ATCCTACCATACAGAAAGACGTTCTTGAGTGTAATGTGAAAACTACCGAAGTTGTAGGAGACATTATACT TAAACCAGCAAATAATAGTTTAAAAATTACAGAAGAGGTTGGCCACACAGATCTAATGGCTGCTTATGTA GACAATTCTAGTCTTACTATTAAGAAACCTAATGAATTATCTAGAGTATTAGGTTTGAAAACCCTTGCTA CTCATGGTTTAGCTGCTGTTAATAGTGTCCCTTGGGATACTATAGCTAATTATGCTAAGCCTTTTCTTAA CAAAGTTGTTAGTACAACTACTAACATAGTTACACGGTGTTTAAACCGTGTTTGTACTAATTATATGCCT TATTTCTTTACTTTATTGCTACAATTGTGTACTTTTACTAGAAGTACAAATTCTAGAATTAAAGCATCTA TGCCGACTACTATAGCAAAGAATACTGTTAAGAGTGTCGGTAAATTTTGTCTAGAGGCTTCATTTAATTA TTTGAAGTCACCTAATTTTCTAAACTGATAAATATTATAATTTGGTTTTTACTATTAAGTGTTTGCCTA GGTTCTTTAATCTACTCAACCGCTGCTTTAGGTGTTTTAATGTCTAATTTAGGCATGCCTTCTTACTGTA CTGGTTACAGAGAAGGCTATTTGAACTCTACTAATGTCACTATTGCAACCTACTGTACTGGTTCTATACC TTGTAGTGTTTGTCTTAGTGGTTTAGATTCTTTAGACACCTATCCTTCTTTAGAAACTATACAAATTACC ATTTCATCTTTTAAATGGGATTTAACTGCTTTTGGCTTAGTTGCAGAGTGGTTTTTGGCATATATTCTTT TCACTAGGTTTTTCTATGTACTTGGATTGGCTGCAATCATGCAATTGTTTTTCAGCTATTTTGCAGTACA TTTTATTAGTAATTCTTGGCTTATGTGGTTAATAATTAATCTTGTACAAATGGCCCCGATTTCAGCTATG GTTAGAATGTACATCTTCTTTGCATCATTTTATTATGTATGGAAAAGTTATGTGCATGTTGTAGACGGTT GTAATTCATCAACTTGTATGATGTGTTACAAACGTAATAGAGCAACAAGAGTCGAATGTACAACTATTGT TAATGGTGTTAGAAGGTCCTTTTATGTCTATGCTAATGGAGGTAAAGGCTTTTGCAAACTACACAATTGG AATTGTGTTAATTTGATACATTCTGTGCTGGTAGTACATTTATTAGTGATGAAGTTGCGAGAGACTTGT CACTACAGTTTAAAAGACCAATAAATCCTACTGACCAGTCTTCTTACATCGTTGATAGTGTTACAGTGAA GAATGGTTCCATCCATCTTTACTTTGATAAAGCTGGTCAAAAGACTTATGAAAGACATTCTCTCTCTCAT TTTGTTAACTTAGACAACCTGAGAGCTAATAACACTAAAGGTTCATTGCCTATTAATGTTATAGTTTTTG ATGGTAAATCAAAATGTGAAGAATCATCTGCAAAATCAGCGTCTGTTTACTACAGTCAGCTTATGTGTCA ACCTATACTGTTACTAGATCAGGCATTAGTGTCTGATGTTGGTGATAGTGCGGAAGTTGCAGTTAAAATG TTTGATGCTTACGTTAATACGTTTTCATCAACTTTTAACGTACCAATGGAAAAACTCAAAACACTAGTTG CAACTGCAGAAGCTGAACTTGCAAAGAATGTGTCCTTAGACAATGTCTTATCTACTTTTATTTCAGCAGC 
TCGGCAAGGGTTTGTTGATTCAGATGTAGAAACTAAAGATGTTGTTGAATGTCTTAAATTGTCACATCAA TCTGACATAGAAGTTACTGGCGATAGTTGTAATAACTATATGCTCACCTATAACAAAGTTGAAAACATGA CACCCCGTGACCTTGGTGCTTGTATTGACTGTAGTGCGCGTCATATTAATGCGCAGGTAGCAAAAAGTCA CAACATTGCTTTGATATGGAACGTTAAAGATTCATGTCATTGTCTGAACAACTACGAAAACAAATACGT AGTGCTGCTAAAAAGAATAACTTACCTTTTAAGTTGACATGTGCAACTACTAGACAAGTTGTTAATGTTG TAACAACAAAGATAGCACTTAAGGGTGGTAAAATTGTTAATAATTGGTTGAAGCAGTTAATTAAAGTTAC ACTTGTGTTCCTTTTTGTTGCTGCTATTTTCTATTTAATAACACCTGTTCATGTCATGTCTAAACATACT GACTTTTCAAGTGAAATCATAGGATACAAGGCTATTGATGGTGGTGTCACTCGTGACATAGCATCTACAG ATACTTGTTTTGCTAACAAACATGCTGATTTTGACACATGGTTTAGCCAGCGTGGTGGTAGTTATACTAA TGACAAAGCTTGCCCATTGATTGCTGCAGTCATAACAAGAGAAGTGGGTTTTGTCGTGCCTGGTTTGCCT GGCACGATATTACGCACAACTAATGGTGACTTTTTGCATTTCTTACCTAGAGTTTTTAGTGCAGTTGGTA ACATCTGTTACACACCATCAAAACTTATAGAGTACACTGACTTTGCAACATCAGCTTGTGTTTTGGCTGC TGAATGTACAATTTTAAAGATGCTTCTGGTAAGCCAGTACCATATTGTTATGATACCAATGTACTAGAA GGTTCTGTTGCTTATGAAAGTTTACGCCCTGACACACGTTATGTGCTCATGGATGGCTCTATTATTCAAT TTCCTAACACCTACCTTGAAGGTTCTGTTAGAGTGGTAACAACTTTTGATTCTGAGTACTGTAGGCACGG CACTTGTGAAAGATCAGAAGCTGGTGTTTGTGTATCTACTAGTGGTAGATGGGTACTTAACAATGATTAT TACAGATCTTTACCAGGAGTTTTCTGTGGTTAGATGCTGTAAATTACTTACTAATATGTTTACACCAC TAATTCAACCTATTGGTGCTTTGGACATATCAGCATCTATAGTAGCTGGTGGTATTGTAGCTATCGTAGT AACATGCCTTGCCTACTATTTTATGAGGTTTAGAAGAGCTTTTGGTGAATACAGTCATGTAGTTGCCTTT AАтАстTTACTATTCCTTATGTCATTCACTGTACTCTGTTTAACACCAGTTTACTCATTCTTACCTGGTG TTTATTCTGTTATTTACTTGTACTTGACATTTTATCTTACTAATGATGTTTCTTTTTTAGCACATATTCA GTGGATGGTTATGTTCACACCTTTAGTACCTTTCTGGATAACAATTGCTTATATCATTTGTATTTCCACA AAGCATTTCTATTGGTTCTTTAGTAATTACCTAAAGAGACGTGTAGTCTTTAATGGTGTTTCCTTTAGTA CTTTTGAAGAAGCTGCGCTGTGCACCTTTTTGTTAAATAAAGAAATGTATCTAAAGTTGCGTAGTGATGT GCTATTACCTCTTACGCAATATAATAGATACTTAGCTCTTTATAATAAGTACAAGTATTTTAGTGGAGCA ATGGATACAACTAGCTACAGAGAAGCTGCTTGTTGTCATCTCGCAAAGGCTCTCAATGACTTCAGTAACT CAGGTTCTGATGTTCTTTACCAACCACCACAAACCTCTATCACCTCAGCTGTTTTGCAGAGTGGTTTTAG AAAAATGGCATTCCCATCTGGTAAAGTTGAGGGTTGTATGGTACAAGTAACTTGTGGTACAACTACACTT AACGGTCTTTGGCTTGATGACGTAGTTTACTGTCCAAGACATGTGATCTGCACCTCTGAAGACATGCTTA ACCCTAATTATGAAGATTTACTCATTCGTAAGTCTAATCATAATTTCTTGGTACAGGCTGGTAATGTTCA ACTCAGGGTTATTGGACATTCTATGCAAAATTGTGTACTTAAGCTTAAGGTTGATACAGCCAATCCTAAG ACACCTAAGTATAAGTTTGTTCGCATTCAACCAGGACAGACTTTTTCAGTGTTAGCTTGTTACAATGGTT CACCATCTGGTGTTTACCAATGTGCTATGAGGCCCAATTTCACTATTAAGGGTTCATTCCTTAATGGTTC ATGTGGTAGTGTTGGTTTTAACATAGATTATGACTGTGTCTCTTTTTGTTACATGCACCATATGGAATTA CCAACTGGAGTTCATGCTGGCACAGACTTAGAAGGTAACTTTTATGGACCTTTTGTTGACAGGCAAACAG CACAAGCAGCTGGTACGGACACAACTATTACAGTTAATGTTTTAGCTTGGTTGTACGCTGCTGTTATAAA TGGAGACAGGTGGTTTCTCAATCGATTTACCACAACTCTTAATGACTTTAACCTTGTGGCTATGAAGTAC AATTATGAACCTCTAACACAAGACCATGTTGACATACTAGGACCTCTTTCTGCTCAAACTGGAATTGCCG TTTTAGATATGTGTGCTTCATTAAAAGAATACTGCAAAATGGTATGAATGGACGTACCATATTGGGTAG TGCTTTATTAGAAGATGAATTACACCTTTTGATGTGTTAGACAATGCTCAGGTGTTACTTTCCAAAGT GCAGTGAAAAGAACAATCAAGGGTACACACCACTGGTTGTTACTCACAATTTTGACTTCACTTTTAGTTT TAGTCCAGAGTACTCAATGGTCTTTGTTCTTTTTTTTGTATGAAAATGCCTTTTTACCTTTTGCTATGGG TATTATTGCTATGTCTGCTTTTGCAATGATGTTTGTCAAACATAAGCATGCATTTCTCTGTTTGTTTTTG TTACCTTCTCTTGCCACTGTAGCTTATTTTAATATGGTCTATATGCCTGCTAGTTGGGTGATGCGTATTA TGACATGGTTGGATATGGTTGATACTAGTTTGTCTGGTTTTAAGCTAAAAGACTGTGTTATGTATGCATC AGCTGTAGTGTTACTAATCCTTATGACAGCAAGAACTGTGTATGATGATGGTGCTAGGAGAGTGTGGACA СTTATGAATGTCTTGACACTCGTTTATAAAGTTTATTATGGTAATGCTTTAGATCAAGCCATTTCCATGT GGGCTCTTATAATCTCTGTTACTTCTAACTACTCAGGTGTAGTTACAACTGTCATGTTTTTGGCCAGAGG TATTGTTTTATGTGTGTTGAGTATTGCCCTATTTTCTTCATAACTGGTAATACACTTCAGTGTATAATG CTAGTTTATTGTTTCTTAGGCTATTTTTGTACTTGTTACTTTGGCCTCTTTTGTTTACTCAACCGCTACT TTAGACTGACTCTTGGTGTTTATGATTACTTAGTTTCTACACAGGAGTTTAGATATATGAATTCACAGGG ACTACTCCCACCCAAGAATAGCATAGATGCCTTCAAACTCAACATTAAATTGTTGGGTGTTGGTGGCAAA CCTTGTATCAAAGTAGCCACTGTACAGTCTAAAATGTCAGATGTAAAGTGCACATCAGTAGTCTTACTCT CAGTTTTGCAACAACTCAGAGTAGAATCATCATCTAAATTGTGGGCTCAATGTGTCCAGTTACACAATGA CATTCTCTTAGCTAAAGATACTACTGAAGCCTTTGAAAAAATGGTTTCACTACTTTCTGTTTTGCTTTCC ATGCAGGGTGCTGTAgACATAAACAAGCTTTGTGAAgAAATGCTGGACAACAGGgCAACCTTACAAGCTA TAGCCTCAGAGTTTAGTTCCCTTCCATCATATGCAGCTTTTGCTACTGCTCAAGAAGCTTATGAGCAGGC TGTTGCTAATGGTGATCTGAAGTTGTTCTTAAAAAGTTGAAGAAGTCTTTGAATGTGGCTAAATCTGAA 
TTTGACCGTGATGCAGCCATGCAACGTAAGTTGGAAAAGATGGCTGATCAAGCTATGACCCAAATGTATA AACAGGCTAGATCTGAGGACAAGAGGGCAAAAGTTACTAGTGCTATGCAGACAATGCTTTTCACTATGCT TAGAAAGTTGGATAATGATGCACTCAACAACATTATCAACAATGCAAGAGATGGTTGTGTTCCCTTGAAC ATAATACCTCTTACAACAGCAGCCAAACTAATGGTTGTCATACCAGACTATAACACATATAAAAATACGT GTGATGGTACAACATTTACTTATGCATCAGCATTGTGGGAAATCCAACAGGTTGTAGATGCAGATAGTAA AATTGTTCAACTTAGTGAAATTAGTATGGACAATTCACCTAATTTAGCATGGCCTCTTATTGTAACAGCT TTAAGGGCCAATTCTGCTGTCAAATTACAGAATAATGAGCTTAGTCCTGTTGCACTACGACAGATGTCTT GTGCTGCCGGTACTACACAAACTGCTTGCACTGATGACAATGCGTTAGCTTACTACAACACAACAAAGGG AGGTAGGTTTGTACTTGCACTGTTATCCGATTTACAGGATTTGAAATGGGCTAGATTCCCTAAGAGTGAT GGAACTGGTACTATCTATACAGAACTGGAACCACCTTGTAGGTTTGTTACAGACACACCTAAAGGTCCTA AAGTGAAgTATTTATACTTTATTAAAGGATTAAACAACCTAAATAGAGGTATGGTACTTGGTAGTTTAGC TGCCACAGTACGTCTACAAGCTGGTAATGCAACAGAAGTGCCTGCCAATTCAACTGTATTATCTTTCTGT GCTTTTGCTGTAGATGCTGCTAAAGCTTACAAAGATTATCTAGCTAGTGGGGGACAACCAATCACTAATT GTGTTAAGATGTTGTGTACACACACTGGTACTGGTCAGGCAATAACAGTTACACCGGAAGCCAATATGGA TCAAGAATCCTTTGGTGGTGCATCGTGTTGTCTGTACTGCCGTTGCCACATAGATCATCCAAATCCTAAA GGATTTTGTGACTTAAAAGGTAAGTATGTACAAATACCTACAACTTGTGCTAATGACCCTGTGGGTTTTA CACTTAAAAACACAGTCTGTACCGTCTGCGGTATGTGGAAAGGTTATGGCTGTAGTTGTGATCAACTCCG CGAACCCATGCTTCAGTCAGCTGATGCACAATCGTTTTTAAACCGGGTTTGCGGTGTAAGTGCAGCCCGT CTTACACCGTGCGGCACAGGCACTAGTACTGATGTCGTATACAGGGCTTTTGACATCTACAATGATAAAG TAGCTGGTTTTGCTAAATTCTAAAAACTAATTGTTGTCGCTTCCAAGAAAAGGACGAAGATGACAATTT AATTGATTCTTACTTTGTAGTTAAGAGACACACTTTCTCTAACTACCAACATGAAGAAACAATTTATAAT TTACTTAAGGATTGTCAGCTGTTGCTAAACATGACTTCTTTAAGTTTAGAATAGACGGTGACATGGTAC CACATATATCACGTCAACGTCTTACTAAATACACAATGGCAGACCTCGTCTATGCTTTAAGGCATTTTGA TGAAGGTAATTGTGACACATTAAAAGAAATACTTGTCACATACAATTGTTGTGATGATGATTATTTCAAT AAAAAGGACTGGTATGATTTTGTAGAAAACCCAGATATATTACGCGTATACGCCAACTTAGGTGAACGTG TACGCCAAGCTTTGTTAAAAACAGTACAATTCTGTGATGCCATGCGAAATGCTGGTATTGTTGGTGTACT GACATTAGATAATCAAGATCTCAATGGTAACTGGTATGATTTCGGTGATTTCATACAAACCACGCCAGGT AGTGGAGTTCCTGTTGTAGATTCTTATTATTCATTGTTAATGCCTATATTAACCTTGACCAGGGCTTTAA CTGCAGAGTCACATGTTGACACTGACTTAACAAAGCCTTACATTAAGTGGGATTTGTTAAAATATGACTT CACGGAAGAGAGGTTAAAACTCTTTGACCGTTATTTAAATATTGGGATCAGACATACCACCCAAATTGT GTTAACTGTTTGGATGACAGATGCATTCTGCATTGTGCAAACTTTAATGTTTTATTCTCTACAGTGTTCC CACTTACAAGTTTTGGACCACTAGTGAGAAAAATATTTGTTGATGGTGTTCCATTTGTAGTTTCAACTGG ATACCACTTCAGAGAGCTAGGTGTTGTACATAATCAGGATGTAAACTTACATAGCTCTAGACTTAGTTTT AAGGAATTACTTGTGTATGCTGCTGACCCTGCTATGCACGCTGCTTCTGGTAATCTATTACTAGATAAAC GCACTACGTGCTTTTCAGTAGCTGCACTTACTAACAATGTTGCTTTTCAAACTGTCAAACCCGGTAATTT TAACAAAGACTTCTATGACTTTGCTGTGTCTAAGGGTTTCTTTAAGGAAGGAAGTTCTGTTGAATTAAAA CACTTCTTCTTTGCTCAGGATGGTAATGCTGCTATCAGCGATTATGACTACTATCGTTATAATCTACCAA CAATGTGTGATATCAGACAACTACTATTTGTAGTTGAAGTTGTTGATAAGTACTTTGATTGTTACGATGG TGGCTGTATTAATGCTAACCAAGTCATCGTCAACAACCTAGACAAATCAGCTGGTTTTCCATTTAATAAA TGGGGTAAGGCTAGACTTTATTATGATTCAATGAGTTATGAGGATCAAGATGCACTTTTCGCATATACAA AACGTAATGTCATCCCTACTATAACTCAAATGAATCTTAAGTATGCCATTAGTGCAAAGAATAGAGCTCG CACCGTAGCTGGTGTCTCTATCTGTAGTACTATGACCAATAGACAGTTTCATCAAAAATTATTGAAATCA ATAGCCGCCACTAGAGGAGCTACTGTAGTAATTGGAACAAGCAAATTCTATGGTGGTTGGCACAACATGT TAAAAACTGTTTATAGTGATGTAGAAAACCCTCACCTTATGGGTTGGGATTATCCTAAATGTGATAGAGC CATGCCTAACATGCTTAGAATTATGGCCTCACTTGTTCTTGCTCGCAAACATACAACGTGTTGTAGCTTG TCACACCGTTTCTATAGATTAGCTAATGAGTGTGCTCAAGTATTGAGTGAAATGGTCATGTGTGGCGGTT CACTATATGTTAAACCAGGTGGAACCTCATCAGGAGATGCCACAACTGCTTATGCTAATAGTGTTTTTAA CATTTGTCAAGCTGTCACGGCCAATGTTAATGCACTTTTATCTACTGATGGTAACAAAATTGCCGATAAG TATGTCCGCAATTTACAACACAGACTTTATGAGTGTCTCTATAGAAATAGAGATGTTGACACAGACTTTG TGAATGAGTTTTACGCATATTTGCGTAAACATTTCTCAATGATGATACTCTCTGACGATGCTGTTGTGTG TTTCAATAGCACTTATGCATCTCAAGGTCTAGTGGCTAGCATAAAGAACTTTAAGTCAGTTCTTTATTAT CAAAACAATGTTTTTATGTCTGAAGCAAAATGTTGGACTGAGACTGACCTTACTAAAGGACCTCATGAAT TTTGCTCTCAACATACAATGCTAGTTAAACAGGGTGATGATTATGTGTACCTTCCTTACCCAGATCCATC AAGAATCCTAGGgGCCGGCTGTTTTGTAGATGATATCGTAAAAACAGATGGTACACTTATGATTGAACGG TTCGTGTCTTTAGCTATAGATGCTTACCCACTTACTAAACATCCTAATCAGGAGTATGCTGATGTCTTTC ATTTGTACTTACAATACATAAGAAAGCTACATGATGAGTTAACAGGACACATGTTAGACATGTATTCTGT TATGCTTACTAATGATAACACTTCAAGGTATTGGGAACCTGAGTTTTATGAGGCTATGTACACACCGCAT ACAGTCTTACAGGCTGTTGGGGCTTGTGTTCTTTGCAATTCACAGACTTCATTAAGATGTGGTGCTTGCA 
TACGTAGACCATTCTTATGTTGTAAATGCTGTTACGACCATGTCATATCAACATCACATAAATTAGTCTT GTCTGTTAATCCGTATGTTTGCAATGCTCCAGGTTGTGATGTCACAGATGTGACTCAACTTTACTTAGGA GGTATGAGCTATTATTGTAAATCACATAAACCACCCATTAGTTTTCCATTGTGTGCTAATGGACAAGTTT TTGGTTTATATAAAAATACATGTGTTGGTAGCGATAATGTTACTGACTTTAATGCAATTGCAACATGTGA CTGGACAAATGCTGGTGATTACATTTTAGCTAACACCTGTACTGAAAGACTCAAGCTTTTTGCAGCAGAA ACGCTCAAAGCTACTGAGGAGACATTTAAACTGTCTTATGGTATTGCTACTGTACGTGAAGTGCTGTCTG ACAGAGAATTACATCTTTCATGGGAAGTTGGTAAACCTAGACCACCACTTAACCGAAATTATGTCTTTAC TGGTTATCGTGTAACTAAAAACAGTAAAGTACAAATAGGAGAGTACACCTTTGAAAAAGGTGACTATGGT GATGCTGTTGTTTACCGAGGTACAACAACTTACAAATTAAATGTTGGTGATTATTTTGTGCTGACATCAC ATACAGTAATGCCATTAAGTGCACCTACACTAGTGCCACAAGAGCACTATGTTAGAATTACTGGCTTATA CCCAACACTCAATATCTCAGATGAGTTTTCTAGCAATGTTGCAAATTATCAAAAGGTTGGTATGCAAAAG TATTCTACACTCCAGGGACCACCTGGTACTGGTAAGAGTCATTTTGCTATTGGCCTAGCTCTCTACTACC CTTCTGCTCGCATAGTGTATACAGCTTGCTCTCATGCCGCTGTTGATGCACTATGTGAGAAGGCATTAAA ATATTTGCCTATAGATAAATGTAGTAGAATTATACCTGCACGTGCTCGTGTAGAGTGTTTTGATAAATTC AAAGTGAATTCAACATTAGAACAGTATGTCTTTTGTACTGTAAATGCATTGCCTGAGACGACAGCAGATA TAGTTGTCTTTGATGAAATTCAATGGCCACAAATATGATTTGAGTGTTGTCAATGCCAGATTACGTGC TAAGCACTATGTGTACATTGGCGACCCTGCTCAATTACCTGCACCACGCACATTGCTAACTAAGGGCACA CTAGAACCAGAATATTCAATTCAGTGTGTAGACTTATGAAAACTATAGGTCCAGACATGTTCCTCGGAA CTTGTCGGCGTTGTCCTGCTGAAATTGTTGACACTGTGAGTGCTTTGGTTTATGATAATAAGCTTAAAGC ACATAAAGACAAATCAGCTCAATGCTTTAAAATGTTTTATAAGGGTGTTATCACGCATGATGTTTCATCT GCAATTAACAGGCCACAAATAGGCGTGGTAAGAGAATTCCTTACACGTAACCCTGCTTGGAGAAAAGCTG TCTTTATTTCACCTTATAATTCACAGAATGCTGTAGCCTCAAAGATTTTGGGACTACCAACTCAAACTGT TGATTCATCACAGGGCTCAGAATATGACTATGTCATATTCACTCAAACCACTGAAACAGCTCACTCTTGT AATGTAAACAGATTTAATGTTGCTATTACCAGAGCAAAAGTAGGCATACTTTGCATAATGTCTGATAGAG ACCTTTATGACAAGTTGCAATTTACAAGTCTTGAAATTCCACGTAGGAATGTGGCAACTTTACAAGCTGA AAATGTAACAGGACTCTTTAAAGATTGTAGTAAGGTAATCACTGGGTTACATCCTACACAGGCACCTACA CACCTCAGTGTTGACACTAAATTAAAACTGAAGGTTTATGTGTTGACATACCTGGCATACCTAAGGACA TGACCTATAGAAGACTCATCTCTATGATGGGTTTTAAAATGAATTATCAAGTTAATGGTTACCCTAACAT GTTTATCACCCGCGAAGAAGCTATAAGACATGTACGTGCATGGATTGGCTTCGATGTCGAGGGGTGTCAT GCTACTAGAGAAGCTGTTGGTACCAATTTACCTTTACAGCTAGGTTTTTCTACAGGTGTTAACCTAGTTG CTGTACCTACAGGTTATGTTGATACACCTAATAATACAGATTTTTCCAGAGTTAGTGCTAAACCACCGCC TGGAGATCAATTTAAACACCTCATACCACTTATGTACAAAGGACTTCCTTGGAATGTAGTGCGTATAAAG ATTGTACAAATGTTAAGTGACACACTTAAAAATCTCTCTGACAGAGTCGTATTTGTCTTATGGGCACATG GCTTTGAGTTGACATCTATGAAGTATTTTGTGAAAATAGGACCTGAGCGCACCTGTTGTCTATGTGATAG ACGTGCCACATGCTTTTCCACTGCTTCAGACACTTATGCCTGTTGGCATCATTCTATTGGATTTGATTAC GTCTATAATCCGTTTATGATTGATGTTCAACAATGGGGTTTTACAGGTAACCTACAAAGCAACCATGATC TGTATTGTCAAGTCCATGGTAATGCACATGTAGCTAGTTGTGATGCAATCATGACTAGGTGTCTAGCTGT CCACGAGTGCTTTGTTAAGCGTGTTGACTGGACTATTGAATATCCTATAATTGGTGATGAACTGAAGATT AATGCGGCTTGTAGAAAGGTTCAACACATGGTTGTTAAAGCTGCATTATTAGCAGACAAATTCCCAGTTC TTCACGACATTGGTAACCCTAAAGCTATTAAGTGTGTACCTCAAGCTGATGTAGAATGGAAGTTCTATGA TGCACAGCCTTGTAGTGACAAAGCTTATAAAATAGAAGAATTATTCTATTCTTATGCCACACATTCTGAC AAATTCACAGATGGTGTATGCCTATTTTGAATTGCAATGTCGATAGATATCCTGCTAATTCCATTGTTT GTAGATTTGACACTAGAGTGCTATCTAACCTTAACTTGCCTGGTTGTGATGGTGGCAGTTTGTATGTAAA TAAACATGCATTCCACACACCAGCTTTTGATAAAAGTGCTTTTGTTAATTTAAAACAATTACCATTTTTC TATTACTCTGACAGTCCATGTGAGTCTCATGGAAAACAAGTAGTGTCAGATATAGATTATGTACCACTAA AGTCTGCTACGTGTATAACACGTTGCAATTTAGGTGGTGCTGTCTGTAGACATCATGCTAATGAGTACAG ATTGTATCTCGATGCTTATAACATGATGATCTCAGCTGGCTTTAGCTTGTGGGTTTACAAACAATTTGAT ACTTATAACCTCTGGAACACTTTTACAAGACTTCAGAGTTTAGAAAATGTGGCTTTTAATGTTGTAAATA AgGGACACTTTGATGGACAACAGgGTGAAGTACCAGTTTCTATCATTAATAACACTGTTTACACAAAAGT TGATGGTGTTGATGTAGAATTGTTTGAAAATAAAACAACATTACCTGTTAATGTAGCATTTGAGCTTTGG GCTAAGCGCAACATTAAACCAGTACCAGAGGTGAAAATACTCAATAATTTGGGTGTGGACATTGCTGCTA ATACTGTGATCTGGGACTACAAAAGAGATGCTCCAGCACATATATCTACTATTGGTGTTTGTTCTATGAC TGACATAGCCAAGAAACCAACTGAAACGATTTGTGCACCACTCACTGTCTTTTTTGATGGTAGAGTTGAT GGTCAAGTAGACTTATTTAGAAATGCCCGTAATGGTGTTCTTATTACAGAAGGTAGTGTTAAAGGTTTAC AACCATCTGTAGGTCCCAAACAAGCTAGTCTTAATGGAGTCACATTAATTGGAGAAGCCGTAAAAACACA GTTCAATTATTATAAGAAAGTTGATGGTGTTGTCCAACAATTACCTGAAACTTACTTTACTCAGAGTAGA AATTTACAAGAATTTAAACCCAGGAGTCAAATGGAAATTGATTTCTTAGAATTAGCTATGGATGAATTCA TTGAACGGTATAAATTAGAAGGCTATGCCTTCGAACATATCGTTTATGGAGATTTTAGTCATAGTCAGTT 
AGGTGGTTTACATCTACTGATTGGACTAGCTAAACGTTTTAAGGAATCACCTTTTGAATTAGAAGATTTT ATTCCTATGGACAGTACAGTTAAAAACTATTTCATAACAGATGCGCAAACAGGTTCATCTAAGTGTGTGT GTTCTGTTATTGATTTATTACTTGATGATTTTGTTGAAATAATAAAATCCCAAGATTTATCTGTAGTTTC TAAGGTTGTCAAAGTGACTATTGACTATACAGAAATTCATTTATGCTTTGGTGTAAAGATGGCCATGTA GAAACATTTTACCCAAAATTACAATCTAGTCAAGCGTGGCAACCGGGTGTTGCTATGCCTAATCTTTACA AAATGCAAAGAATGCTATTAGAAAAGTGTGACCTTCAAAATTATGGTGATAGTGCAACATTACCTAAAGG CATAATGATGAATGTCGCAAAATATACTCAACTGTGTCAATATTTAAACACATTAACATTAGCTGTACCC TATAATATGAGAGTTATACATTTTGGTGCTGGTTCTGATAAAGGAGTTGCACCAGGTACAGCTGTTTTAA GACAGTGGTTGCCTACGGGTACGCTGCTTGTCGATTCAGATCTTAATGACTTTGTCTCTGATGCAGATTC AACTTTGATTGGTGATTGTGCAACTGTACATACAGCTAATAAATGGGATCTCATTATTAGTGATATGTAC GACCCTAAGACTAAAAATGTTACAAAAGAAAATGACTCTAAAGAGGGTTTTTTCACTTACATTTGTGGGT TTATACAACAAAAGCTAGCTCTTGGAGGTTCCGTGGCTATAAAGATAACAGAACATTCTTGGAATGCTGA TCTTTATAAGCTCATGGGACACTTCGCATGGTGGACAGCCTTTGTTACTAATGTGAATGCGTCATCATCT GAAGCATTTTTAATTGGATGTAATTATCTTGGCAAACCACGCGAACAAATAGATGGTTATGTCATGCATG CAAATTACATATTTGGAGGAATACAAATCCAATTCAGTTGTCTTCCTATTCTTTATTTGACATGAGTAA ATTTCCCCTTAAATTAAGGGGTACTGCTGTTATGTCTTTAAAAGAAGGTCAAATCAATGATATGATTTTA TCTCTTCTTAGTAAAGGTAGACTTATAATTAGAGAAAACAACAGAGTTGTTATTTCTAGTGATGTTCTTG TTAACAACTAA

Figure 4. Example of the complete sequence of the hCoV-19/Jordan.

in consonance with previous reports that stated that clade G apparently originated from Shanghai before it was imported into European countries [17] [20] [21].

\section{Conclusion}

The results of this study show conclusively a unique pandemic profile of multiple geographical sources of COVID-19 importation into Jordan primarily through UK, Belgium, Sweden, Russia and Morocco, which are entry points into Jordan. This is also due to the reciprocal heavy air traffic route between Jordan and European capitals. It can be inferred based on the identical homology (99.98\% - 100\%) of the 28 sequences of hCoV-19/Jordan strain that there was no marked change in the virus mutation that occurred during the period of this study.

\section{Acknowledgements}

The authors would like to acknowledge the generous support of Professor Kristian Andersen (Scripps Research Institute, TSRI, La Jolla, CA, USA) and his joint-permission with the Biolab Diagnostic Laboratory, Jordan (personal communication) to use the SARS-CoV-2 sequences from Jordan in this paper. The authors also would like to acknowledge the use of the data and the bioinformatics software available on the Public Domains of both NCBI GeneBank (http://www.ncbi.nlm.nih.gov/) and the Global Initiative on Sharing All Influenza Data (GISAID) (http://www.gisaid.org/). The authors would like to acknowledge the efforts of the Ministry of Health (http://www.moh.gov.jo), Government of Jordan for the financial support and the valuable efforts on administrating the National Surveillances Program (as set by the National orders numbers; 17 -20) including the samples collections of the SARS-CoV-2). 


\section{Data Availability}

All the 28 sequences of the hCoV-19/Jordan used in this paper have been used as available on the public Domains in both NCBI GeneBank

(http://www.ncbi.nlm.nih.gov/) and the Global Initiative on Sharing All Influenza Data (GISAID) (http://www.gisaid.org/) under the name of hCoV-19/ Jordan as per each respective accession number. All clinical data of the patients, epidemiological details as well as sampling surveillances were maintained in the National record of the Ministry of Health of Jordan.

\section{Conflicts of Interest}

The authors declare no conflicts of interest regarding the publication of this paper.

\section{References}

[1] Koyama, T., Platt, D. and Parida, L. (2020) Variant Analysis of SARS-CoV-2 Genomes. Bulletin of the World Health Organization, 98, 495-504.

https://www.who.int/docs/default-source/coronaviruse/situation-reports/20200410sitrep-81-covid-19 https://doi.org/10.2471/BLT.20.253591

[2] Lu, R., Zhao, X., Li, J., Niu, P., Yang, B., Wu, H., Wang, W., Song, H., Huang, B., Zhu, N., Bi, Y., Ma, X., Zhan, F., Wang, L., Hu, T., Zhou, H., Hu, Z., Zhou, W., Zhao, L., Chen, J., Meng, Y., Wang, J., Lin, Y., Yuan, J., Xie, Z., Ma, J., Liu, W.J., Wang, D., Xu, W., Holmes, E.C., Gao, G.F., Wu, G., Chen, W., Shi, W. and Tan, W. (2020) Genomic Characterization and Epidemiology of 2019 Novel Coronavirus: Implications for Virus Origins and Receptor Binding. The Lancet, 395, 565-574. https://doi.org/10.1016/S0140-6736(20)30251-8

[3] Zhou, P., Yang, X., Wang, X., et al. (2020) A Pneumonia Outbreak Associated with a New Coronavirus of Probable Bat Origin. Nature, 579, 270-273. https://doi.org/10.1038/s41586-020-2012-7

[4] Cavanagh, D. (2007) Coronavirus Avian Infectious Bronchitis Virus. Veterinary Research, 38, 281-297. https://doi.org/10.1051/vetres:2006055

[5] Ismail, M.M., Tang, A.Y. and Saif, Y.M. (2003) Pathogenicity of Turkey Coronavirus in Turkeys and Chickens. Avian Diseases, 47, 515-522. https://doi.org/10.1637/5917

[6] Moira, C.-Y. and Xu, R.H. (2003) SARS: Epidemiology. Respirology, 8, S9-S14. https://doi.org/10.1046/j.1440-1843.2003.00518.x

[7] Corman, V.M., Müller, M.A., Costabel, U., Timm, J., Binger, T., Meyer, B., et al. (2012) Assays for Laboratory Confirmation of Novel Human Coronavirus (hCoVEMC) Infections. Eurosurveillance, 17, 20334. https://doi.org/10.2807/ese.17.49.20334-en

[8] Zaki, A.M., van Boheemen, S., Bestebroer, T.M., Osterhaus, A.D. and Fouchier, R.A. (2012) Isolation of a Novel Coronavirus from a Man with Pneumonia in Saudi Arabia. The New England Journal of Medicine, 367, 1814-1820. https://doi.org/10.1056/NEJMoa1211721

[9] Lee, J., Chowell, G. and Jung, E. (2016) A Dynamic Compartmental Model for the Middle East Respiratory Syndrome Outbreak in the Republic of Korea: A Retrospective Analysis on Control Interventions and Superspreading Events. Journal of 
Theoretical Biology, 408, 118-126. https://doi.org/10.1016/j.jtbi.2016.08.009

[10] Chung, E.H., Kim, Y.-J., Chung, E.H., Kim, D.-W., et al. (2017) The Clinical and Virological Features of the First Imported Case Causing MERS-CoV Outbreak in South Korea, 2015. BMC Infectious Diseases, 17, Article No. 498. https://doi.org/10.1186/s12879-017-2576-5

[11] Andersen, K.G., Rambaut, A., Lipkin, W.I., Holmes, E.C. and Garry, R.F. (2020) The Proximal Origin of SARS-CoV-2. Nature Medicine, 26, 450-452.

http://www.nature.com/naturemedicine https://doi.org/10.1038/s41591-020-0820-9

[12] Corman, V.M., Landt, O., Kaiser, M., Molenkamp, R., Meijer, A., Chu, D.K.W., Bleicker, T., Brünink, S., Schneider, J., Schmidt, M.L., Mulders, D.G.J.C., Haagmans, B.L., van der Veer, B., van den Brink, S., Wijsman, L., Goderski, G., Romette, J.-L., Ellis, J., Zambon, M., Peiris, M., Goossens, H., Reusken, C., Koopmans, M.P.G. and Drosten, C. (2020) Detection of 2019 Novel Coronavirus (2019-nCoV) by Real-Time RT-PCR. Eurosurveillance, 25, Article ID: 2000045. https://doi.org/10.2807/1560-7917.ES.2020.25.3.2000045

[13] Forster, P., Forster, L., Renfrew, C. and Forster, M. (2020) Phylogenetic Network Analysis of SARS-CoV-2 Genomes. Proceedings of the National Academy of Sciences of the United States of America, 117, 9241-9243.

https://doi.org/10.1073/pnas.2004999117

[14] Guan, Q., Sadykov, M., Mfrrej, S., Hala, S., Naeem, R., Nugmanova, R., Al-Omari, A., Salih, S., Al-Mutair, A., Carr, M.J., Hall, W.W., Arold, S.T. and Pain, A. (2020) A Genetic Barcode of SARS-CoV-2 for Monitoring Global Distribution of Different Clades during the COVID-19 Pandemic. International Journal of Infectious Diseases, 100, 216-223. https://doi.org/10.1016/j.ijid.2020.08.052

[15] Gu, W., Miller, S. and Chiu, C.Y. (2019) Clinical Metagenomic Next-Generation Sequencing for Pathogen Detection. Annual Review of Pathology: Mechanisms of Disease, 14, 319-338. https://doi.org/10.1146/annurev-pathmechdis-012418-012751

[16] Mercatelli, D. and Giorgi, F.M. (2020) Geographic and Genomic Distribution of SARS-CoV-2 Mutations. Frontiers in Microbiology, 11, 1-13. https://doi.org/10.3389/fmicb.2020.01800

[17] Zehender, G., Lai, A., Bergna, A., Meroni, L., Riva, A., Balotta, C., Tarkowski, M., Gabrieli, A., Bernacchia, D., Rusconi, S., Rizzardini, G., Antinori, S. and Galli, M. (2020) Genomic Characterization and Phylogenetic Analysis of SARS-COV-2 in Italy. Journal of Medical Virology. https://doi.org/10.1101/2020.03.15.20032870

[18] World Health Organization (WHO) (2020) Coronavirus Disease 2019 (COVID-19) Situation Report-81.

https://www.who.int/docs/default-source/coronaviruse/situation-reports/20200410sitrep-81-covid-19

[19] European Centre for Disease Control and Prevention (ECDC) (2020) COVID-19 Situation update Worldwide, as of 10 April 2020.

https://www.ecdc.europa.eu/en/geographical-distribution-2019-ncov-cases

[20] Bartolini, B., Rueca, M., Gruber, C., Messina, F., Carletti, F., Giombini, E., et al. (2020) SARS-CoV-2 Phylogenetic Analysis, Lazio Region, Italy, February-March 2020. Emerging Infectious Diseases, 26, 1842-1845. https://doi.org/10.3201/eid2608.201525

[21] Giovanetti, M., Angeletti, S., Benvenuto, D. and Ciccozzi, M. (2020) A Doubt of Multiple Introduction of SARS-CoV-2 in Italy: A Preliminary Overview. Journal of Medical Virology, 92, 1634-1636. https://doi.org/10.1002/jmv.25773 